\title{
Compliance with severe sepsis bundles and its effect on patient outcomes of severe community-acquired pneumonia in a limited resources country
}

Qi Guo ${ }^{1,2}$, Hai-Yan Li³, Yi-Min Li², Ling-Bo Nong², Yuan-Da Xu², Guo-Qing He², Xiao-Qing Liu², Mei Jiang'2 ${ }^{2}$ Zheng-Iun $\mathrm{XiaO}^{2}$, Nan-Shan Zhong ${ }^{2}$

\author{
'Department of Respiratory Medicine, Affiliated Futian Hospital, Guangdong Medical \\ College, Shenzhen, Guangdong, China \\ ${ }^{2}$ Guangzhou Institute of Respiratory Diseases (State Key Laboratory of Respiratory \\ Diseases), First Affiliated Hospital, Guangzhou Medical University, Guangzhou, \\ Guangdong, China \\ ${ }^{3}$ Department of Primary Care, Affiliated Futian Hospital, Guangdong Medical College, \\ Shenzhen, Guangdong, China
}

Submitted: 27 June 2012

Accepted: 24 October 2012

Arch Med Sci 2014; 10, 5: 970-978

DOI: 10.5114 /aoms.2014.46216

Copyright @ 2014 Termedia \& Banach

\section{Abstract}

Introduction: Validation of compliance with severe sepsis bundles is still needed. The purpose of this study was to determine compliance and its outcomes in severe community-acquired pneumonia (CAP) patients in a limited resources country.

Material and methods: A prospective cohort study of 212 severe CAP patients was carried out. The implementation programme was organized into two continuous phases. The primary outcomes were compliance and hospital mortality.

Results: Compliance with administration of antibiotics and vasopressors as well as plateau pressure on average $<30 \mathrm{~cm} \mathrm{H} \mathrm{H}_{2} \mathrm{O}$ was high in both groups. In the bundles group, patients received more serum lactate monitoring $(62.3 \%$ vs. $11.3 \%)$, more blood cultures ( $47.1 \%$ vs. $24.5 \%)$, more fluid resuscitation $(63.2 \%$ vs. $26.4 \%)$ and volumes infused $(1319.8 \pm 1107.4 \mathrm{ml} \mathrm{vs.} 461.9 \pm 799.3 \mathrm{ml})$, more inotropic dobutamine and/or packed red blood cells $(21.7 \%$ vs. $10.0 \%)$, more low-dose steroids (56.5\% vs. $15.0 \%)$, and more glucose control $(51.9 \%$ vs. $6.6 \%)$ compared with such patients in the control group. The rates of total compliance with 6-hour, 24-hour, and 6/24-hour bundles in the prospective period were $47.1 \%, 51.9 \%$, and $42.5 \%$, respectively. Hospital mortality was reduced from $44.3 \%$ to $29.2 \%(p=0.023)$ in the bundles group, and the compliant subgroup had a more than twofold decrease in mortality $(17.8 \%$ vs. $37.7 \%, p=0.003$ ). Serum lactate measured, blood cultures, and fluid resuscitation showed independent relationships with decreased mortality.

Conclusions: Total compliance was relatively low, but the implementation of severe sepsis bundles could clearly reduce mortality from severe CAP.

Key words: severe sepsis bundles, severe community-acquired pneumonia, severe sepsis, septic shock, compliance, mortality.

\section{Introduction}

The mortality rate due to pneumonia has shown little improvement over time despite advances in antimicrobial therapy and improved intensive care medicine [1-3]. It was as high as $58 \%$ when patients with se-
Corresponding author: Qi Guo MD, PhD Guangdong Medical College 518033 Shenzhen, China Phone: +86 13923714808 E-mail: qiguo007@sina.com 
vere community-acquired pneumonia (CAP) were admitted to the intensive care unit (ICU) [4].

The Surviving Sepsis Campaign (SSC) was launched in 2002 as a collaborative initiative of three international organizations. The initial guidelines were published in 2004 and an updated version in 2008. Its objective was, through the development and promulgation of evidence-based guidelines that facilitated the application of knowledge derived from clinical trials to bedside practice, to effect a $25 \%$ reduction in the relative risk of death from severe sepsis and septic shock $[5,6]$. The development and publication of guidelines often do not lead to changes in clinical behaviour and guidelines are rarely, if ever, integrated into bedside practice in a timely fashion. The SSC undertook a broad educational programme to implement them by integrating their recommendations into resuscitation and management bundles. The feasibility of implementing severe sepsis bundles was validated in the United States, Europe and South America and as a result the mortality from severe sepsis was reduced [7-19]. Compliance with the bundles and its impact were heterogeneous among institutions and countries [20]. Therefore, the primary evidence obtained so far lacks enough substance and quality to promote the guidelines as a global standard of care for universal applicability. Further evaluations and primary studies are still needed [21], especially in countries with limited resources.

There has only been one report so far in English of compliance with the bundles in Asia [22]. The study did not aim to evaluate the impact of the sepsis protocol during a one-month observation period. As the centres included in this study might not be fully representative, achievement rates reported might overestimate or underestimate the true degree of compliance with recommended care and should be interpreted with caution. On the other hand, the healthcare systems in many Asian countries differ greatly from those in the above-mentioned developed countries. Therefore, further studies are still warranted in Asia.

Pneumonia is the major source of infection in severe sepsis patients in our respiratory ICU (RICU). Therefore, we decided to study this population. Adult patients who suffered from severe CAP with severe sepsis or septic shock were enrolled in this study to determine compliance with severe sepsis bundles and to report its effect on patient outcomes in a limited resources country.

\section{Material and methods}

\section{Design and setting}

A prospective observational cohort study from 1 November 2006 to 31 July 2008 was carried out on adult patients who met criteria for severe CAP with severe sepsis or septic shock presenting to the RICU in a Chinese affiliated tertiary hospital of a medical college. Similar patients presenting between 1 November 2004 and 31 October 2006 were used as historical controls.

\section{Criteria for enrolment}

The CAP was defined as an acute infection of the pulmonary parenchyma associated with an acute infiltrate on the chest radiograph with two or more symptoms including fever $\left(>38^{\circ} \mathrm{C}\right)$, hypothermia $\left(<36^{\circ} \mathrm{C}\right)$, rigors, sweats, new cough or change in colour of respiratory secretions, chest discomfort or dyspnoea. Severe CAP with severe sepsis or septic shock was defined when one of two major criteria (the need for invasive mechanical ventilation or vasopressors) was fulfilled. Septic shock was defined as systolic arterial pressure (SAP) $<90 \mathrm{~mm} \mathrm{Hg}$, mean arterial pressure (MAP) $<60 \mathrm{~mm} \mathrm{Hg}$, or a reduction in SAP of $>40 \mathrm{~mm} \mathrm{Hg}$ from baseline, which was persistent, despite adequate fluid resuscitation, in the absence of other causes for hypotension [3, 23, 24].

Exclusion criteria were patients who: a) had a cardiac arrest, or b) had end-stage chronic disease (malignancy, chronic renal failure, chronic obstructive pulmonary disease, or acquired immune deficiency syndrome). Patients with severe heart failure, with neoplastic disease (but not in the end stage), with severe asthma, with chronic renal failure stage I, or with brain stroke were also enrolled.

\section{Components of severe sepsis bundles}

Six-hour and 24-hour severe sepsis bundles adapted from the SSC standard sepsis resuscitation and management bundles are listed in Table I [25]. Since the use of recombinant human activated protein $C$ is not approved in China, it was eliminated from the 24-hour severe sepsis bundle.

\section{Implementation of severe sepsis bundles}

The 21-month implementation programme was organized into two continuous phases that incorporated education and intervention. During the education phase (in November 2006), conference lectures were delivered to all physicians and nurses in the RICU and the emergence department (ED) on the SSC, severe CAP definitions and recognition, haemodynamic monitoring, and the initiation and components of the bundles. Timely initiation and completion of the bundles were emphasized. Time zero was defined as the point when the diagnosis of severe CAP was established in the ED. Serum lactate measured, blood cultures obtained prior to antibiotic administration, and initial empirical antibiotics 
Table I. Severe sepsis bundles

\section{6-hour severe sepsis bundle:}

1. Serum lactate measured.

2. Blood cultures obtained prior to antibiotic administration.

3. Initial empirical antibiotics administered within $1 \mathrm{~h}$.

4. In the event of hypotension and/or serum lactate $>4 \mathrm{mmol} / \mathrm{l}$ :

(a) Deliver an initial minimum of $20 \mathrm{ml} / \mathrm{kg}$ of crystalloid (or colloid equivalent: $1.1 \mathrm{ml} / \mathrm{kg}$ of $20 \%$ albumin or $4.8 \mathrm{ml} / \mathrm{kg}$ of $6 \%$ hydroxyethyl starch).

(b) Apply vasopressors (noradrenaline or dopamine) for hypotension not responding to initial fluid resuscitation to maintain mean arterial pressure $(\mathrm{MAP}) \geq 65 \mathrm{~mm} \mathrm{Hg}$.

5. In the event of persistent septic shock and/or serum lactate $>4 \mathrm{mmol} / \mathrm{l}$, administration of inotropic dobutamine and/or transfusion of packed red blood cells (when haemoglobin $(\mathrm{Hb})<70 \mathrm{~g} / \mathrm{l}$ ) to achieve a central venous pressure (CVP) of $\geq 8 \mathrm{~mm} \mathrm{Hg}$ and central venous oxygen saturation $\left(\mathrm{ScvO}_{2}\right)$ of $\geq 70 \%$.

24-hour severe sepsis bundle:

1. Low dosage corticosteroid (40-60 mg/day of methylprednisolone) administered for septic shock requiring continued infusion of vasopressors.

2. Glucose control maintained $<8.3 \mathrm{mmol} / \mathrm{l}$.

3. For mechanically ventilated patients maintain inspiratory plateau pressures $<30 \mathrm{~cm} \mathrm{H} \mathrm{H}_{2} \mathrm{O}$.

administered within $1 \mathrm{~h}$ were considered once severe CAP was diagnosed, and the admission was fulfilled through an Emergency Path. Insulin was intravenously administered by infusion pump to maintain blood glucose level $<8.3 \mathrm{mmol} / \mathrm{l}$. In the intervention phase (between 1 December 2006 and 31 July 2008), the personnel were issued with a personal pocket information card as a daily reminder of the processes involved in the delivery of the bundles and weekly feedback was provided by the senior physicians to all personnel in order to optimize compliance. The feedback incorporated oral information, cards posted in the RICU and the ED, and group discussions.

\section{Definition of total compliance}

On the basis of severity of illness, implementation of all necessary components of 6-hour, 24-hour, or 6/24-hour severe sepsis bundles was defined as total compliance. Had some components been unnecessary on the basis of the SSC, a patient would still have been regarded as receiving all components as long as the remaining ones were implemented.

\section{Approval of study design}

The study was approved by our Institutional Review Boards (Review Board of Guangdong Medical College and Review Board of Guangzhou Medical University). Ethical approval from the regulation committee (Ethical Committee of Guangzhou) was granted for the study protocol with a waiver of informed consent.

\section{Outcomes}

The primary outcomes were compliance and hospital mortality. Secondary outcomes incorporated ventilator-free days estimated from the ICU length of stay and parameters at $72 \mathrm{~h}$ after start of therapy. The endpoints for evaluation were referred to Kortgen's study [9]. Laboratory variables were measured by the hospital clinical laboratory.

\section{Grouping and data collection}

One hundred and thirteen patients were enrolled in the cohort study period. Seven cases were excluded from this study according to the exclusion criteria. The remaining 106 patients, 46 (43.4\%) patients who suffered from septic, shock were enrolled in the bundles group, which was further divided into the compliant and the non-compliant subgroups at the end of the study according to total compliance with 6/24-hour severe sepsis bundles. One hundred and six discharged patients who were selected through the formal case-control method to match all baseline characteristics of the patients in the prospective group were assigned to the control group. A senior physician was in charge of collecting the data. Demographics, co-morbid conditions, vital signs, laboratory data, and compliance data were collected. The volumes of $6 \%$ hydroxyethyl starch and $20 \%$ albumin were converted into equivalent dosages of crystalloid [26]. Severity-of-illness scores were calculated, including the acute physiology and chronic health evaluation (APACHE) II scores and sequential organ failure assessment (SOFA) scores. Missing values were assumed to be normal [27]. Indeed, patients with less severe illness were more likely to have missing values for laboratory findings. The statistician was blinded to the study and study hypothesis.

\section{Statistical analysis}

All statistical analyses were performed with Statistical Package for the Social Science for Windows version 13.0 (SPSS, Chicago, IL, USA). Categorical variables and continuous data were reported as percentages and mean \pm standard deviation (SD), respectively. Chi-square $\left(\chi^{2}\right)$ test and independent-samples $t$ test were used when appropriate in testing for differences between groups. Univariate and multivariate logistic regression analyses were employed. All components of severe sepsis bundles were entered into a multivariate logistic regression model. Statistical significance was defined as $p<0.05$. 


\section{Results}

\section{Baseline characteristics of patients}

No significant differences were observed between the two patient groups with respect to baseline characteristics (Table II). The aetiologies were not detected in many patients in the control group. Hence, the data were not shown. The initial antibiotic regimens in the two groups were consistent with the guidelines on the management of CAP $[3,28]$, in addition to subsequently cultured pathogens. All patients in the bundles group had central venous pressure (CVP) and central venous oxygen saturation $\left(\mathrm{ScvO}_{2}\right)$ measured and the av-

Table II. Baseline characteristics of patients (mean \pm SD, $n=212$ )

\begin{tabular}{|c|c|c|c|c|}
\hline Variable & Control group & Bundles group & Value of $\chi^{2}$ or $t$ & Value of $p$ \\
\hline Age [years] & $62.8 \pm 14.2$ & $64.0 \pm 15.2$ & 0.941 & 0.348 \\
\hline Male sex (\%) & 77.4 & 66.1 & 3.347 & 0.067 \\
\hline Body temperature $\left[{ }^{\circ} \mathrm{C}\right]$ & $37.6 \pm 1.0$ & $37.8 \pm 1.3$ & 1.485 & 0.149 \\
\hline Heart rate [beats/min] & $113.6 \pm 22.4$ & $116.4 \pm 27.6$ & 0.827 & 0.409 \\
\hline Respiratory rate [breaths/min] & $29.0 \pm 6.7$ & $29.9 \pm 10.0$ & 0.780 & 0.436 \\
\hline MAP [mm Hg] & $81.1 \pm 18.2$ & $81.0 \pm 19.9$ & 0.035 & 0.972 \\
\hline Blood glucose $[\mathrm{mmol} / \mathrm{l}]$ & $9.0 \pm 2.6$ & $9.1 \pm 3.3$ & 0.248 & 0.804 \\
\hline White blood cell count $\left[10^{9} / I\right]$ & $13.6 \pm 7.5$ & $15.0 \pm 6.6$ & 1.532 & 0.127 \\
\hline $\mathrm{PaO}_{2} / \mathrm{FIO}_{2}[\mathrm{~mm} \mathrm{Hg}]$ & $141.5 \pm 42.9$ & $150.2 \pm 58.4$ & 1.236 & 0.218 \\
\hline Serum creatinine $[\mu \mathrm{mol} / \mathrm{l}]$ & $106.4 \pm 53.9$ & $111.7 \pm 79.6$ & 0.568 & 0.571 \\
\hline Total bilirubin $[\mu \mathrm{mol} / \mathrm{l}]$ & $25.3 \pm 38.9$ & $29.0 \pm 69.9$ & 0.469 & 0.639 \\
\hline Alanine aminotransferase [U/I] & $88.0 \pm 155.5$ & $58.7 \pm 115.0$ & 1.559 & 0.121 \\
\hline Platelets $\left[10^{9} / 1\right]$ & $211.7 \pm 108.7$ & $205.1 \pm 94.4$ & 0.470 & 0.639 \\
\hline Haematocrit (\%) & $33.6 \pm 6.4$ & $32.9 \pm 6.5$ & 0.815 & 0.416 \\
\hline Serum lactate $[\mathrm{mmol} / \mathrm{l}]$ & $2.8 \pm 1.4$ & $3.1 \pm 1.5$ & 0.748 & 0.465 \\
\hline APACHE II score & $15.4 \pm 5.3$ & $15.9 \pm 7.5$ & 0.991 & 0.317 \\
\hline SOFA score & $6.1 \pm 3.4$ & $6.9 \pm 4.7$ & 1.464 & 0.145 \\
\hline Shock (\%) & 37.7 & 43.4 & 0.704 & 0.401 \\
\hline \multicolumn{5}{|l|}{ Comorbidities (\%): } \\
\hline Hypertension & 38.4 & 40.6 & 0.265 & 0.529 \\
\hline Coronary heart disease & 11.3 & 6.6 & 1.445 & 0.229 \\
\hline Heart failure & 12.3 & 10.4 & 0.188 & 0.665 \\
\hline $\begin{array}{l}\text { Chronic obstructive pulmonary } \\
\text { disease }\end{array}$ & 26.4 & 33.0 & 1.107 & 0.293 \\
\hline Diabetes mellitus & 14.2 & 18.9 & 0.856 & 0.355 \\
\hline Liver disease & 7.5 & 9.4 & 0.243 & 0.622 \\
\hline Tumour & 19.8 & 11.3 & 2.907 & 0.088 \\
\hline Nervous system disease & 7.5 & 9.4 & 0.243 & 0.622 \\
\hline Chronic renal insufficiency & 7.5 & 5.7 & 0.306 & 0.580 \\
\hline Alcohol abuse (\%) & 5.7 & 1.9 & 2.078 & 0.149 \\
\hline Smoking (\%) & 34.9 & 35.8 & 0.021 & 0.886 \\
\hline $\begin{array}{l}\text { Duration to establish severe CAP } \\
\text { diagnosis [min] }\end{array}$ & $61.8 \pm 57.2$ & $60.3 \pm 49.5$ & 0.253 & 0.712 \\
\hline \multicolumn{5}{|l|}{ Antibiotic therapy: } \\
\hline Antibiotics adequate (\%) & 93.7 & 95.8 & 0.319 & 0.527 \\
\hline Duration [days] & $12.6 \pm 14.3$ & $12.9 \pm 15.1$ & 0.237 & 0.872 \\
\hline
\end{tabular}

$\mathrm{PaO}_{2} / \mathrm{FlO}_{2}$ - arterial oxygen pressure/fraction inspired oxygen. 


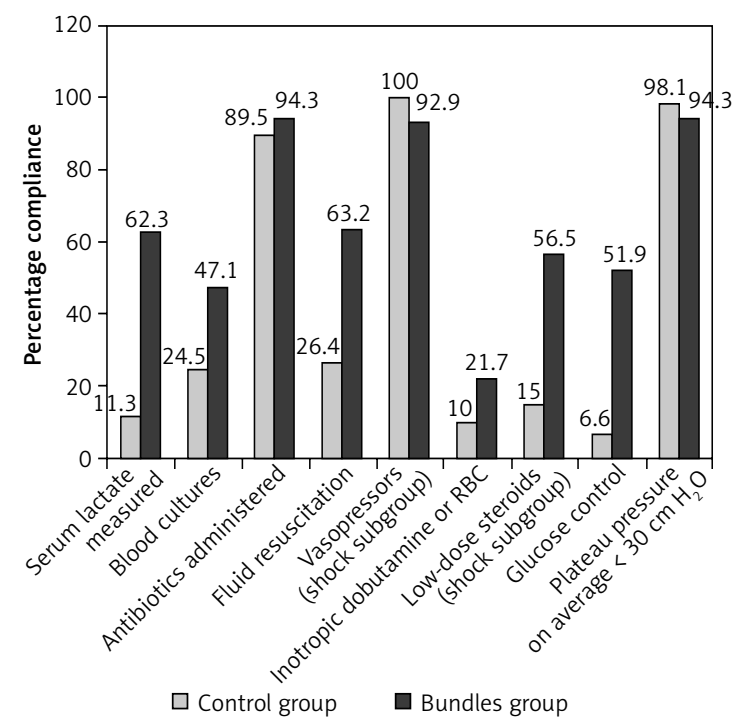

Figure 1. Compliance with components of severe sepsis bundles $(n=212)$

$R B C$ - red blood cells

erages were $9.4 \pm 1.7 \mathrm{~mm} \mathrm{Hg}$ and $68.3 \pm 9.8 \%$, respectively. However, these parameters were not measured in most patients in the control group. Therefore, the comparison could not be drawn.

\section{Compliance with severe sepsis bundles}

Compliance with administration of antibiotics within $1 \mathrm{~h}$, administration of vasopressors (if necessary), and plateau pressure on average < 30 $\mathrm{cm} \mathrm{H}_{2} \mathrm{O}$ was high in both groups ( $p, 0.206,0.085$, 0.149 , respectively; Figure 1$)$. Significant differences were found between the two groups with respect to serum lactate measured, blood cultures obtained prior to antibiotic administration, initiation of fluid resuscitation and volumes infused, administration of inotropic dobutamine and/or transfusion of packed red blood cells, low-dose steroids (if necessary), and glucose control $(p,<$ $0.001,0.001,<0.001,0.005$, < 0.001, and <0.001, respectively; Figure 1). Inotropic dobutamine was administered to 17 patients, 11 patients simultaneously received an average 4-unit transfusion of packed red cells in the bundles group, whereas the numbers in the control group were 4, 2, and 2 , respectively. The average volume infused within $6 \mathrm{~h}$ in the bundles group was more than that in the control group $(1319.8 \pm 1107.4 \mathrm{ml}$ vs. $461.9 \pm 799.3$ $\mathrm{ml} ; p<0.001)$. The rates of total compliance with 6-hour, 24-hour, and 6/24-hour bundles in the bundles group were $47.1 \%, 51.9 \%$, and $42.5 \%$, whereas the rates in the control group were $0.0 \%$, $0.0 \%$, and $0.0 \%(p,<0.001,<0.001$, and $<0.001)$, respectively.

\section{Outcomes at 72 hours}

Significant differences were observed between the two groups in respiratory rate, MAP, blood glucose, arterial oxygen pressure/fraction inspired oxygen $\left(\mathrm{PaO}_{2} / \mathrm{FIO}_{2}\right)$, serum creatinine, serum lactate, APACHE II scores, and SOFA scores at $72 \mathrm{~h}$ after the start of therapy (Table III). Central venous pressure increased significantly from $9.4 \pm 1.7 \mathrm{~mm} \mathrm{Hg}$ at baseline to $10.9 \pm 1.8 \mathrm{~mm} \mathrm{Hg}$ at $72 \mathrm{~h}$ after the start of therapy in the bundles group ( $p<$ 0.001 ), as did $\mathrm{ScvO}_{2}$ during this time period (68.3 $\pm 9.8 \%$ vs. $79.5 \pm 3.8 \%, p<0.001$ ).

Table III. Outcomes at $72 \mathrm{~h}($ mean \pm SD, $n=212)$

\begin{tabular}{|c|c|c|c|c|}
\hline Parameter & Control group & Bundles group & Value of $t$ & Value of $p$ \\
\hline Body temperature $\left[{ }^{\circ} \mathrm{C}\right]$ & $37.2 \pm 1.0$ & $37.3 \pm 0.9$ & 0.236 & 0.814 \\
\hline Heart rate [beats/min] & $100.3 \pm 21.5$ & $99.7 \pm 17.5$ & 0.214 & 0.831 \\
\hline Respiratory rate [breaths/min] & $27.7 \pm 7.0$ & $25.2 \pm 7.6$ & 2.542 & 0.012 \\
\hline MAP [mm Hg] & $87.8 \pm 12.9$ & $91.4 \pm 10.6$ & 2.185 & 0.030 \\
\hline Blood glucose $[\mathrm{mmol} / \mathrm{l}]$ & $8.9 \pm 2.3$ & $7.4 \pm 1.8$ & 5.360 & $<0.001$ \\
\hline White blood cell count $\left[10^{9} / 1\right]$ & $13.0 \pm 8.9$ & $14.2 \pm 7.5$ & 1.034 & 0.302 \\
\hline $\mathrm{PaO}_{2} / \mathrm{FlO}_{2}[\mathrm{~mm} \mathrm{Hg}]$ & $172.4 \pm 101.6$ & $223.1 \pm 83.3$ & 3.971 & $<0.001$ \\
\hline Serum creatinine $[\mu \mathrm{mol} / \mathrm{l}]$ & $118.3 \pm 87.5$ & $97.0 \pm 52.6$ & 2.145 & 0.033 \\
\hline Total bilirubin $[\mu \mathrm{mol} / \mathrm{l}]$ & $33.7 \pm 64.9$ & $35.7 \pm 98.9$ & 0.179 & 0.858 \\
\hline Alanine aminotransferase [U/I] & $254.8 \pm 1219.2$ & $51.2 \pm 50.9$ & 1.718 & 0.087 \\
\hline Platelets $\left[10^{9} / I\right]$ & $159.6 \pm 109.8$ & $162.0 \pm 112.3$ & 0.161 & 0.872 \\
\hline Haematocrit (\%) & $30.7 \pm 5.4$ & $29.8 \pm 5.3$ & 1.229 & 0.221 \\
\hline Serum lactate [mmol/l] & $4.1 \pm 3.9$ & $2.5 \pm 0.7$ & 2.979 & 0.004 \\
\hline APACHE II score & $11.3 \pm 5.7$ & $9.5 \pm 4.7$ & 2.637 & 0.009 \\
\hline SOFA score & $6.6 \pm 4.1$ & $5.4 \pm 4.3$ & 2.170 & 0.031 \\
\hline
\end{tabular}


Table IV. Baseline characteristics of patients and mortality in the bundles group according to total compliance (mean \pm SD, $n=106)$

\begin{tabular}{|lcccc|}
\hline Parameter & $\begin{array}{c}\text { Compliant subgroup } \\
(n=45)\end{array}$ & $\begin{array}{c}\text { Non-compliant } \\
\text { subgroup }(n=61)\end{array}$ & Value of $\chi^{2}$ or $t$ & Value of $p$ \\
\hline Age [years] & $61.7 \pm 19.2$ & $61.4 \pm 15.5$ & 0.096 & 0.924 \\
\hline Male sex (\%) & 68.9 & 63.9 & 0.283 & 0.594 \\
\hline MAP [mm Hg] & $81.6 \pm 21.0$ & $80.5 \pm 19.2$ & 0.289 & 0.773 \\
\hline White blood cell count $\left[10^{9} / 1\right]$ & $16.5 \pm 7.4$ & $14.0 \pm 5.7$ & 1.926 & 1.460 \\
\hline PaO $/$ FIO 2 [mm Hg] & $145.3 \pm 63.2$ & $162.1 \pm 52.2$ & 1.206 & 0.148 \\
\hline APACHE II score & $16.9 \pm 7.3$ & $15.2 \pm 7.6$ & 1.470 & 0.231 \\
\hline SOFA score & $7.4 \pm 5.3$ & $6.0 \pm 4.0$ & 1.969 & 0.146 \\
\hline Shock $(\%)$ & 44.4 & 31.1 & 4.970 & 0.161 \\
\hline Mortality, $n(\%)$ & $8(17.8)$ & $23(37.7)$ & 026 \\
\hline
\end{tabular}

\section{Hospital mortality and ventilator-free days}

Hospital mortality in patients enrolled in the bundles group compared with that in the control group was $29.2 \%$ vs. $44.3 \%\left(\chi^{2}=5.192, p=0.023\right)$, reflecting a $15.1 \%$ absolute reduction in mortality. There was a significantly decreased odds ratio (OR) for mortality in patients who received bundles treatment $(\mathrm{OR}=0.393 ; 95 \%$ confidence interval $(\mathrm{Cl}), 0.209-0.741 ; p=0.004)$. A multivariate logistic regression analysis was performed. Serum lactate measured, blood cultures obtained prior to antibiotic administration, and fluid resuscitation showed independent relationships with decreased mortality $(\mathrm{OR}=0.112,0.459$, and $0.247 ; 95 \% \mathrm{Cl}$ : 0.037-0.334, 0.211-0.997, and 0.091-0.635; $p$, $<0.001,0.039$, and $<0.001$; respectively).

The two subgroups were comparable with respect to age, gender, and severity at baseline (Table IV). Compared with the non-compliant subgroup, the compliant subgroup had a more than twofold decrease in mortality $(17.8 \%$ vs. $37.7 \%$. $\mathrm{OR}=0.222 ; 95 \% \mathrm{Cl}: 0.082-0.602 ; p=0.003)$.

All patients in both groups were mechanically ventilated. In the bundles group, patients had significant longer ventilator-free days compared with such patients in the control group (6.1 \pm 6.7 vs. 3.9 \pm 6.6 days; $p=0.017$ ).

\section{Discussion}

Compliance with administration of antibiotics and vasopressors and plateau pressure on average $<30 \mathrm{~cm} \mathrm{H} \mathrm{H}_{2} \mathrm{O}$ was high. The rates of total compliance with the bundles in the prospective period increased significantly. Hospital mortalities were significantly reduced in the bundles group and in the compliant subgroup. Serum lactate measurement, blood cultures, and fluid resuscitation were independently associated with decreased mortality.

Initial empirical antibiotics were generally administered within $1 \mathrm{~h}$, suggesting that our phy- sicians know and readily accept the concept of the association between timing of antibiotic administration and outcomes [29-31]. Nguyen et al. [32] found that physicians were more willing to accept hypotension than serum lactate concentration as a criterion to initiate resuscitation, as in the current study; that is to say, some patients with serum lactate $>4 \mathrm{mmol} / \mathrm{l}$ might not receive fluid resuscitation. Therefore, compliance with initiation of fluid resuscitation was not high. Compliance with administration of vasopressors was high in both groups, but the implementation of administration of inotropic dobutamine and/ or transfusion of packed red blood cells was poor (the worst), suggesting that our physicians did not put more emphasis on the imbalance between oxygen consumption and supply when dealing with persistent septic shock. Glucose control was the lowest compliant element of the 24-hour bundle in our study despite continuous insulin infusion. Scrupulous avoidance of iatrogenic hypoglycaemia frequently resulted in blood glucose $>8.3 \mathrm{mmol} / \mathrm{l}$.

Our physicians found that weekly feedback facilitated the implementation of the bundles. Levy et al. [13] also reported that compliance with the entire resuscitation bundle and with the entire management bundle increased to $31.3 \%$ and $36.1 \%$, respectively, by the end of 2 years. The compliance rate with all elements of the sepsis resuscitation bundle increased from $12.7 \%$ at baseline to $37.7 \%$ and $53.7 \%$ during the weekly feedback and sepsis response team activation periods, respectively $(p<0.001)$ [33]. Compliance with a 6-hour severe sepsis bundle at the beginning was low and increased by the end (from $8.1 \%$ to $53.7 \%$ ) [8].

The total compliance was not high in the current study, as was it in Phua's study (compliance rates for the resuscitation and management bundles were $7.6 \%$ and $3.5 \%$, respectively) [22] and Schramm's study (the compliance rate with 
all elements of the sepsis resuscitation bundle increased from $12.7 \%$ at baseline to $37.7 \%$ and $53.7 \%$ during the weekly feedback and sepsis response team activation periods, respectively) [33]. Two of the major factors limiting high total compliance in our study might be lack of sufficient staff in the RICU and the ED and difficulty in maintaining the time intervals. This is likely to be improved by better staff awareness of the benefits and timeliness of implementing the bundles. Levy's report emphasized the importance of early management and was reminiscent of the concept of the golden $6 \mathrm{~h}$ and silver $24 \mathrm{~h}$ for trauma [34].

The SSC has had some impact; however, there is still a long way to go [35]. What about the feasibility of implementing the bundles in undeveloped countries? Our institution is the state key laboratory of respiratory diseases. This might be envisaged to interpret the higher compliance in the current study, compared with that in Phua's multicentre study [22]. It is likely to imply that the implementation of the bundles might be very arduous in many undeveloped countries, especially those in Asia. This might be an important message that would have potential to improve care on a global basis. Hence, future long-term multicentre studies should be performed to assess the compliance in limited resources countries.

The patients without hypotension would still have received fluid resuscitation had serum lactate been measured, which could be of survival benefit. Similarly, obtaining blood cultures may be useful if the blood cultures revealed a pathogen that was not being treated effectively with the antibiotics chosen. If the clinician was then able to act upon that information and correct the antibiotic choice, this could have an impact on mortality. These considerations might explain why serum lactate measured and blood cultures showed independent relationships with decreased mortality.

Our current data revealed the pivotal role of implementing severe sepsis bundles in the management of severe CAP. The $15.1 \%$ absolute hospital mortality reduction in our study was similar to that reported by Nguyen et al. (18.7\%) [8], Kortgen et al. (26\%) [9], Micek et al. (18.3\%) [11], Lefrant et al. (13.0\%) [16], Schramm et al. (8.3\%) [33], and Castellanos-Ortega et al. (19.8\%) [36]. In the compliant subgroup, patients had a more than twofold decrease in mortality compared with such patients in the non-compliant subgroup in the current study. Gao et al. [7] reported that the mortality in the compliant group decreased from $49 \%$ to $23 \%$ ( $p=0.01$ ), compared with the non-compliant group. Mortality was $61.1 \%$ in the non-bundle group versus $20 \%$ with the bundle $(p<0.001)$ [15]. Delivery of the interventions had an association with reduced mortality: for the sepsis six, $20.0 \%$ compared with $44.1 \%(p<0.001)$; for the resuscitation bundle, 5.9\% compared with 51\% $(p<0.001)$ [14].

Several limitations of this study deserve comment. Comparing crude mortality rates before and after an intervention ignores the underlying trend of decreased mortality and might give rise to misleading conclusions. Another limitation is that it was performed only in patients with severe CAP and not other types of sepsis from other sources of infection such as intra-abdominal infections or soft tissue infections. There might be some imbalance in the aetiology of pneumonia between the two groups. The study was not multicentre. Finally, long-term follow-up was not done; therefore, it was uncertain whether the implementation of the bundles and its effect would be similar to what Ferrer et al. [37] reported (during long-term follow-up, compliance with the sepsis resuscitation bundle returned to baseline but compliance with the sepsis management bundle and mortality remained stable with respect to the post-intervention period).

In conclusion, total compliance was relatively low, but the implementation of severe sepsis bundles was associated with a significant decrease in mortality from severe CAP.

\section{Acknowledgments}

Qi Guo and Hai-Yan Li are joint first authors.

The study was funded by the nonprofit foundation of Ying-Dong Huo in Hongkong.

Part of the data was accepted for presentation (Number: P2999) in the Thematic Poster session entitled "Etiology of lower respiratory tract infections and treatment in special situations" at the ERS (European Respiratory Society) Barcelona 2010 Annual Congress (18-22 September).

Q.G, H-Y.L, Y-M.L, Z-L.X and N-S.Z made substantial contributions to the conception and design. Q.G and H-Y.L were in charge of data collection and wrote the manuscript. Y-M.L, L-B.N, Y-D.X, G-Q.H, and X-Q.L made substantial contributions to acquisition of data. M.J was in charge of statistical analysis. Z-L.X and N-S.Z were involved in general supervision of the research group.

We are indebted to the staffing of the ED, and nurses and postgraduates of the RICU for making contributions to this study.

We thank Chris J. Corrigan (King's College London) for assistance in English editing.

\section{References}

1. Feikin DR, Schuchat A, Kolczak M, et al. Mortality from invasive pneumococcal pneumonia in the era of antibiotic resistance, 1995-1997. Am J Public Health 2000; 90: 223-9.

2. American Thoracic Society; Infectious Diseases Society of America. Guidelines for the management of adults with 
hospital-acquired, ventilator-associated, and healthcareassociated pneumonia. Am J Respir Crit Care Med 2005 171: 388-416.

3. Mandell LA, Wunderink RG, Anzueto A, et al. Infectious Diseases Society of America/American Thoracic Society consensus guidelines on the management of community-acquired pneumonia in adults. Clin Infect Dis 2007; 44 (Suppl 2): S27-72.

4. Restrepo MI, Anzueto A. Severe community-acquired pneumonia. Infect Dis Clin North Am 2009; 23: 503-20.

5. Dellinger RP, Carlet JM, Masur $\mathrm{H}$, et al. Surviving sepsis campaign guidelines for management of severe sepsis and septic shock. Crit Care Med 2004; 32: 858-73.

6. Dellinger RP, Levy MM, Carlet JM, et al. Surviving sepsis campaign: international guidelines for management of severe sepsis and septic shock: 2008. Crit Care Med 2008; 36: 296-327.

7. Gao F, Melody T, Daniels DF, Giles S, Fox S. The impact of compliance with 6-hour and 24-hour sepsis bundles on hospital mortality in patients with severe sepsis: a prospective observational study. Crit Care 2005; 9: R764-70.

8. Nguyen HB, Corbett SW, Steele R, et al. Implementation of a bundle of quality indicators for the early management of severe sepsis and septic shock is associated with decreased mortality. Crit Care Med 2007; 35: 1105-12.

9. Kortgen A, Niederprüm P, Bauer M. Implementation of an evidence-based "standard operating procedure" and outcome in septic shock. Crit Care Med 2006; 34: 943-9.

10. Shapiro NI, Howell MD, Talmor D, et al. Implementation and outcomes of the Multiple Urgent Sepsis Therapies (MUST) protocol. Crit Care Med 2006; 34: 1025-32.

11. Micek ST, Roubinian N, Heuring T, et al. Before-after study of a standardized hospital order set for the management of septic shock. Crit Care Med 2006; 34 2707-13.

12. Jones AE, Focht A, Horton JM, Kline JA. Prospective external validation of the clinical effectiveness of an emergency department-based early goal-directed therapy protocol for severe sepsis and septic shock. Chest 2007; 132: 425-32.

13. Levy MM, Dellinger RP, Townsend SR, et al. The surviving sepsis campaign: results of an international guideline-based performance improvement program targeting severe sepsis. Crit Care Med 2010; 38: 367-74.

14. Daniels R, Nutbeam T, McNamara G, Galvin C. The sepsis six and the severe sepsis resuscitation bundle: a prospective observational cohort study. Emerg Med J 2011; 28: 507-12.

15. Patel GW, Roderman N, Gehring H, Saad J, Bartek W. Assessing the effect of the surviving sepsis campaign treatment guidelines on clinical outcomes in a community hospital. Ann Pharmacother 2010; 44: 1733-8.

16. Lefrant JY, Muller L, Raillard A, et al. Reduction of the severe sepsis or septic shock associated mortality by reinforcement of the recommendations bundle: a multicenter study. Ann Fr Anesth Reanim 2010; 29: 621-8.

17. Cardoso T, Carneiro AH, Ribeiro O, Teixeira-Pinto A, Costa-Pereira A. Reducing mortality in severe sepsis with the implementation of a core 6-hour bundle: results from the Portuguese community-acquired sepsis study (SACiUCI study). Crit Care 2010; 14: R83.

18. Pestaña D, Espinosa E, Sangüesa-Molina JR, et al. Com pliance with a sepsis bundle and its effect on intensive care unit mortality in surgical septic shock patients. J Trauma 2010; 69: 1282-7.
19. Shiramizo SC, Marra AR, Durão MS, Paes ÂT, Edmond MB, Pavão dos Santos OF. Decreasing mortality in severe sepsis and septic shock patients by implementing a sepsis bundle in a hospital setting. PLoS One 2011; 6: e26790.

20. Machado FR, Mazza BF. Improving mortality in sepsis: analysis of clinical trials. Shock 2010; 34 (Suppl. 1): S54-S58.

21. Finfer S. The surviving sepsis campaign: robust evaluation and high-quality primary research is still needed. Crit Care Med 2010; 38: 683-4.

22. Phua J, Koh Y, Du B, et al. Management of severe sepsis in patients admitted to Asian intensive care units: prospective cohort study. BMJ 2011; 342: d3245.

23. Phua J, See KC, Chan YH, et al. Validation and clinical implications of the IDSA/ATS minor criteria for severe community-acquired pneumonia. Thorax 2009; 64: 598-603.

24. Levy MM, Fink MP, Marshall JC, et al. 2001 SCCM/ESICM/ ACCP/ATS/SIS International Sepsis Definitions Conference. Crit Care Med 2003; 31: 1250-6.

25. Surviving Sepsis Campaign and Institute for Healthcare Improvement. Sepsis Bundle. Available online at: http:// www.ihi.org/IHI/Topics/CriticalCare/Sepsis/Tools/SevereSepsisBundle.htm. Accessed May 26, 2006.

26. American Thoracic Society. Evidence-based colloid use in the critically ill: American Thoracic Society Consensus Statement. Am J Respir Crit Care Med 2004; 170: 1247-59.

27. Aujesky D, Auble TE, Yealy DM, et al. Prospective comparison of three validated prediction rules for prognosis in community-acquired pneumonia. Am J Med 2005; 118: 384-92.

28. Niederman MS, Mandell LA, Anzueto A, et al. Guidelines for the management of adults with community-acquired pneumonia. Diagnosis, assessment of severity, antimicrobial therapy, and prevention. Am J Respir Crit Care Med 2001; 163: 1730-54.

29. Houck PM, Bratzler DW, Nsa W, Ma A, Bartlett JG. Timing of antibiotic administration and outcomes for Medicare patients hospitalized with community-acquired pneumonia. Arch Intern Med 2004; 164: 637-44.

30. Ferrer R, Artigas A, Suarez D, et al. Effectiveness of treatments for severe sepsis: a prospective, multicenter, observational study. Am J Respir Crit Care Med 2009; 180: 861-6.

31. Gaieski DF, Mikkelsen ME, Band RA, et al. Impact of time to antibiotics on survival in patients with severe sepsis or septic shock in whom early goal-directed therapy was initiated in the emergency department. Crit Care Med 2010; 38: 1045-53.

32. Nguyen HB, Lynch EL, Mou JA, Lyon K, Wittlake WA, Corbett SW. The utility of a quality improvement bundle in bridging the gap between research and standard care in the management of severe sepsis and septic shock in the emergency department. Acad Emerg Med 2007; 14: 1079-86.

33. Schramm GE, Kashyap R, Mullon JJ, Gajic O, Afessa B. Septic shock: a multidisciplinary response team and weekly feedback to clinicians improve the process of care and mortality. Crit Care Med 2011; 39: 252-8.

34. Levy MM, Macias WL, Vincent JL, et al. Early changes in organ function predict eventual survival in severe sepsis. Crit Care Med 2005; 33: 2194-201.

35. Cronshaw HL, Daniels R, Bleetman A, Joynes E, Sheils $M$. Impact of the surviving sepsis campaign on the recognition and management of severe sepsis in the emer- 
gency department: are we failing? Emerg Med J 2011;

28: 670-5.

36. Castellanos-Ortega A, Suberviola B, García-Astudillo LA, et al. Impact of the surviving sepsis campaign protocols on hospital length of stay and mortality in septic shock patients: results of a three-year follow-up quasi-experimental study. Crit Care Med 2010; 38: 1036-43.

37. Ferrer R, Artigas A, Levy MM, et al. Improvement in process of care and outcome after a multicenter severe sepsis educational program in Spain. JAMA 2008; 299: 2294-303. 